\title{
Misdiagnosing sleep disorders as primary psychiatric conditions
}

\author{
Gregory Stores
}

Abstract Sleep disorders are relevant to psychiatric practice in a number of ways, including the possibility that they may be misdiagnosed as fundamentally psychiatric conditions in patients of all ages. This risk exists in a wide range of collectively very common sleep disorders which need to be considered in explaining insomnia, excessive sleepiness or disturbed episodes of behaviour associated with sleep (parasomnias). Examples given include circadian sleep-wake cycle disorders (such as the delayed sleep phase syndrome), obstructive sleep apnoea, narcolepsy, Kleine-Levin syndrome, sleep paralysis and rapid eye movement (REM) sleep behaviour disorder. Failure to recognise and treat such disorders is likely to cause and perpetuate psychological problems. Correct recognition requires familiarity with the range and manifestations of sleep disorders.

There is a compelling argument for viewing sleep and its disorders as a central theme in psychiatry and for clinicians to be familiar with sleep disorder medicine whatever the age of their patients. Sad to say, however, psychiatry (and, for that matter, clinical psychology) generally shares in the neglect of this field in medical and other teaching and training areas.

There are several main points of contact between psychiatry and sleep disorders. The first is that sleep disturbance is a common feature of a wide range of psychiatric disorders in adults (Benca, 2000) and also in children and adolescents (Stores \& Wiggs, 2001). This is well recognised in depression, where clinical abnormalities of sleep are prominent and where disturbed sleep physiology is also a feature (at least, in severe depressive disorders in adults), possibly with aetiological, therapeutic and prognostic implications. Brunello et al (2000) have reviewed these issues, pointing out the various unknowns that need further research.

The various types of sleep disorder which commonly complicate the whole range of psychiatric conditions seem to be less well known. Not only are psychiatric disorders themselves capable of affecting sleep and wakefulness: certain psychotropic medications can have the same effect (Obermeyer \& Benca, 1996), possibly adding to the patient's difficulties. Sleep disturbance can be an early sign of impending psychiatric disorder. A review by Gillin (1998) demonstrates that, in both those with and without a serious psychiatric history, insomnia and/or hypersomnia are risk factors for later development or recurrence of depression, anxiety states or substance misuse. Detection and treatment of the sleep disturbance at an early age might have important preventive value (Ford \& Kamerow, 1989). The significance of sleep disturbance in various forms, not merely insomnia, in psychiatric disorders is illustrated by the recent report of a close association between nightmares and death from suicide in the general population (Tanskanen et al, 2001).

Another important connection between sleep disorders and psychiatry concerns the harmful effects on psychological function of sleep disturbance, especially if it is persistent. This disturbance may take the form of loss of sleep, impaired quality of sleep and/or inappropriate timing of the sleep period. Experimental findings and clinical observation have demonstrated potentially widespread effects on mood, behaviour and performance in adults with no previous psychological problems (Bonnet, 2000). There are increasing reports of comparable effects in children and adolescents (Fallone et al, 2002). These adverse influences on psychological function are considered by many to be particularly serious because sleep disturbance is seemingly so common in modern Westernised societies.

Reports of disturbed psychological function attributable to sleep disturbance are in keeping with the idea that the sleep disturbance may be a contributory factor (and in some cases the basic cause) of actual psychiatric illness. For example, it has been suggested that sleep disturbance has at

Gregory Stores is Professor of Developmental Neuropsychiatry and Fellow of Linacre College in the University of Oxford (University Section, Park Hospital, Old Road, Headington, Oxford OX3 7LQ, UK) and an honorary consultant to the Oxfordshire Mental Healthcare NHS Trust. His main clinical and research interests are sleep and its disorders in children and adults, on which he has published extensively. 
least an ancillary role in some forms of depression. Recently, interest has been growing in the possibility that a primary sleep disturbance may be responsible for some cases of attention-deficit hyperactivity disorder in both children and adults (Walters et al, 2000; Naseem et al, 2001). Rapid eye movement (REM) sleep abnormalities have been implicated by some in a variety of psychiatric disorders, including post-traumatic stress disorder, some forms of schizophrenia and other neuropsychiatric disorders in which psychotic phenomena occur (Howland, 1997). Even in the absence of psychiatric disorders, insomnia has been associated with impaired healthrelated quality of life, similar to that resulting from chronic conditions such as congestive heart failure and depression (Katz \& McHorney, 2002), and with increased use of both general medical and mental health services (Weissman et al, 1997).

The remainder of this article is concerned with a further fundamental link, i.e. the possibility that, because of similarity in their clinical manifestations, sleep disorders may be mistaken for primary psychiatric conditions. The consequences of this are likely to be serious, including delays in the provision of appropriate treatment, worsening of the condition because of delay and disappointment, loss of treatment opportunities altogether or adverse effects of inappropriate medication.

Some of the following examples arise from points already made about the possible psychological effects of persistent sleep disturbance on mental state. Collectively, these and the other examples are drawn from all three major types of complaint described in the International Classification of Sleep Disorders (ICSD; American Sleep Disorders Association, 1997): difficulty getting off to sleep or remaining asleep (insomnia); excessive daytime sleepiness; and episodes of disturbed behaviour or experiences related to sleep (parasomnias). In addition, sleep disorders that are secondary to physical illness or physical disorders will be considered in so far as they might also be mistakenly viewed as essentially psychiatric in origin.

Box 1 describes specific sleep disorders where this risk of misdiagnosis is probably greatest. As well as considering these individual conditions in psychiatric diagnosis, it is important to be aware of the psychiatric effects of sleep disturbance in general. Whatever its specific origin, sleep loss or poor quality sleep commonly leads to changes of mood such as irritability (and even aggression) and depression. Other psychological consequences include complaints of fatigue or other physical symptoms and, in extreme cases, disorientation, perceptual changes and persecutory ideas. Clearly, it is of fundamental clinical importance to distinguish between sleep disturbance as a cause of
Box 1 Specific sleep disorders at risk of misdiagnosis as primary sleep disorders

Delayed sleep phase syndrome

Other circadian-rhythm sleep disorders:

- advanced sleep phase syndrome

- jet lag

- night shift work

- irregular sleep-wake schedule

- non-24-hours sleep-wake syndrome

Obstructive sleep apnoea syndrome

Narcolepsy

Other causes of pathological sleepiness:

- idiopathic hypersomnia

- Kleine-Levin syndrome

Isolated sleep paralysis

REM sleep behaviour disorder

Other sleep disorders associated with abnormal behaviour

- hypnagogic/hypnopompic hallucinations

- hypnic jerks

- sensory sleep starts

- restless legs syndrome

- sleepwalking/sleep terrors

- overlap parasomnia disorder

- sleep-related eating disorders

- nocturnal frontal lobe epilepsy

psychiatric disorders and sleep disturbance as a result of such disorders.

\section{Delayed sleep phase syndrome}

Delayed sleep phase syndrome (DSPS) is the most common of the circadian-rhythm sleep disorders (Wagner, 1990), which are important causes of insomnia and excessive daytime sleepiness. These disorders arise either from malfunction of the endogenous system which regulates the sleep-wake rhythm (the 'biological clock', located in the suprachiasmatic nucleus of the hypothalamus) or from environmental effects on these systems (e.g. in jet lag or shift work). Because they are basically biological in nature, this group of sleep disorders requires treatment to produce physiological readjustment of the basic sleep-wake mechanism (e.g. by means of chronotherapy schedules, bright light therapy or melatonin). Dagan (2002) has reviewed the evidence that psychiatric disturbance (notably personality disorders and depression) is commonly found in adults with circadian-rhythm sleep disorders and that certain psychotropic drugs (haloperidol and fluvoxamine) can disrupt sleepwake cycle mechanisms.

Delayed sleep phase syndrome is thought to occur at all ages but especially in adolescence. For a variety 
of reasons (e.g. bedtime struggles of young children with parents; late-night social activities in adolescents, often involving consumption of sleepdisrupting substances such as caffeine, alcohol or nicotine; entertainment at home into the early hours; or working late, in the case of adults) a pattern is established of not going to sleep until very late. This results in the biological clock resetting itself and a delay of the sleep phase. Consequently, it becomes physiologically impossible to go to sleep earlier, no matter how motivated a person is to do so. After only a few hours of sound sleep, getting up for school or work is very difficult because sleep requirements have not been met, and fatigue, tiredness and the other consequences of being sleepy (irritability and poor concentration, for example) are experienced during the day. Sleeping in very late when able to, especially at weekends (which is characteristic of the condition) maintains the sleep delay.

The basic biological nature of DSPS is easily overlooked. Going to sleep late and having difficulty waking in the morning may well be misconstrued as difficult 'adolescent' behaviour or some other form of psychological shortcoming but entreaties to change have no effect. Persistence of the condition readily gives rise to psychological complications, including depression, which are described in a high proportion of people with DSPS (Regenstein \& Monk, 1995). The situation can be further complicated if alcohol or other substances are used either to get to sleep at night or to keep awake during the day.

Because DSPS does not seem to be well-known to professionals (or indeed the public), it is likely to be misinterpreted and attention paid to resulting complications of the condition rather than the true origins of the disturbed mood or behaviour. As in the other sleep disorders discussed here, recognition and appropriate treatment rest on awareness that DSPS exists and on familiarity with its characteristic features. Treatment can be difficult if the patient is motivated to maintain the abnormal sleep pattern, e.g. as part of school refusal. In such circumstances, wider psychiatric intervention is required.

\section{Other circadian-rhythm sleep disorders}

\section{Advanced sleep phase syndrome}

One of the changes of sleep physiology in later life is the tendency for the timing of the sleep phase to move forward, with the onset of sleep occurring in the evening. This may result in the reverse of DSPS, i.e. a troublesome and potentially socially embarrassing inability to stay awake in the evening, followed by waking early in the morning, by which time enough sleep been obtained (the 'advanced sleep phase syndrome'). The same effect can occur at a younger age, if the child is consistently put to bed very early in the evening, in which case he or she may wake far too early the next day for the rest of the family. The main psychiatric point in adults, perhaps especially the elderly, is that early morning waking originating in this way should not be viewed as a sign of a depressive disorder. Instead of antidepressant measures, the appropriate treatment is to reset the sleep phase to a more socially satisfactory time.

The remaining circadian-rhythm sleep disorders also have psychological effects which need to be distinguished from primary psychiatric disorders.

\section{Jet lag}

The effects of jet lag are usually short lived, but travellers who frequently cross several time zones on each flight can develop chronic sleep disturbances that can have serious effects on mood and performance which are worsened if alcohol, sedatives or stimulants are used in an attempt to alleviate the symptoms.

\section{Night shift work}

Night shift work poses a much greater problem because of the large numbers of people involved. This is partly due to increases in 24-hour consumer services, although occupations such as nursing and police work may inevitably involve shift work. Many (possibly most) shift workers suffer psychological problems as well as physical complaints as a result of trying to be active at night when their biological clock dictates that they should be asleep. Performance levels are commonly affected, with high rates of mistakes and accidents, sometimes with serious consequences. Daytime sleep is often inadequate, being limited in duration and quality. Impaired social relationships, including those between partners, can easily result from the chronic sleep disturbance itself plus the restrictions of shift work patterns on normal family and social life. Again, the situation is not improved if attempts are made at self-treatment using alcohol or other substances. Although a minority seems to adjust satisfactorily to working the night shift, there are many who do not and they need help (including the cooperation of employers) to minimise the physiological and psychological consequences of their work patterns.

\section{Irregular sleep-wake schedule}

Irregular sleep-wake schedule, with disorganised and variable sleep and waking times, can be caused 
by a general lack of consistency and routine in family functioning. This sleep disorder can also be associated with the use of recreational or illicit drugs. Many patients with severe congenital or degenerative brain disorders also show the same disorganised pattern. Sleep is broken up into a number of short periods, inconsistently timed within each 24-hour period. This degree of sleep disruption is very likely to cause further psychological disturbance which is easily mistaken for a primary psychiatric disorder. It can also increase the degree of cognitive or behavioural disturbance of patients with a degenerative or other type of brain disorder.

\section{Non-24-hour sleep-wake syndrome}

The remaining circadian sleep rhythm, the 'non-24hour sleep-wake syndrome', is unusual in the general population. It is mainly described in blind patients, who are deprived of the main cue, i.e. light perception, by which sleep-wake rhythms are established and maintained (or 'entrained'). Damage to or maldevelopment of biological clock mechanisms (usually associated with severe intellectual impairment) or localised hypothalamic dysfunction can have the same effect. Again, help with the psychological problems resulting from such sleep deprivation should be combined with attempts to improve the sleep-wake pattern. However, this can be very difficult.

\section{Obstructive sleep apnoea syndrome}

This common and often serious condition occurs in people of all ages (Ohayon et al, 1997). At least $4 \%$ of middle-aged males, $2 \%$ of adult females and $1 \%$ of children may be affected to some degree but only a small minority seek medical advice. These rates are significantly exceeded in certain groups, including obese people, the elderly, those with hypertension and some forms of learning disability such as Down's syndrome.

The phrase 'obstructive sleep apnoea syndrome' implies repeated interruption of airflow through the upper airway during sleep, in spite of respiratory effort, because of obstruction of the airway. Each interruption is followed by arousal from sleep, which has adverse physical and psychological effects. The main sign of the condition is chronic noisy breathing during sleep, including snoring, associated with apnoeic episodes. In children, compromised respiration and its consequences can occur without apnoeas because the obstruction is usually partial rather than complete. The main risk factor in adults is obesity. In contrast, most childhood cases are caused by enlarged tonsils or adenoids. Although snoring is a feature of the syndrome, many people snore without having the condition. However, if noisy breathing during sleep is combined with daytime sleepiness or underperformance, the possibility of obstructive sleep apnoea syndrome should be considered. Treatment is usually effective in improving sleep and daytime functioning.

The consequences of obstructive sleep apnoea syndrome can be severe, including cardiovascular disease. Similarly, effects on psychological function during the day can be disabling because the repeated interruptions to overnight sleep, which can occur hundreds of times each night, impair its restorative function. The constant nocturnal oxygen desaturations are likely to add to this effect. Daytime sleepiness (including actually falling asleep) is the main feature in adults but, paradoxically, the opposite effect (i.e. overactivity) is reported in children. The sleepiness contributes to a higher risk of car and other accidents and underperformance at work. Children's performance at school may well suffer. Impairments of memory, attention (especially vigilance) and other cognitive deficits have been described (Engelman et al, 2002). The degree of cognitive impairment can be extreme and misinterpreted as a dementing illness of the more usual type. Other psychiatric effects that have been reported are depression, confusional states and psychotic disorders. Various parasomnias can occur, including sudden distressing awakenings at the end of an obstructive event, and these become an additional source of concern. The poor quality of life imposed by obstructive sleep apnoea syndrome, with restrictions on family and social relationships, adds to the direct psychological complications of the condition.

There is a serious risk of misinterpreting these various neuropsychiatric consequences of the syndrome if the presence of the underlying sleeprelated breathing disorder is not realised. For example, depression appears to be one of the conditions for which patients may well be treated before their obstructive sleep apnoea syndrome is correctly diagnosed (Smith et al, 2002). Perhaps particularly in the elderly, failure to recognise the syndrome may lead to missed opportunities to successfully treat intellectual decline (Bliwise, 1996).

\section{Narcolepsy}

This neurological condition (mainly an abnormality of REM sleep) is not the rarity sometimes supposed (Thorpy, 2001). It occurs in 2-5 per 10000 of the population (a similar level to Parkinson's disease or multiple sclerosis) and begins in childhood or adolescence in $30 \%$ or more of cases. The classic tetrad of the full narcolepsy syndrome consists of 
sleep attacks, cataplexy, hypnagogic hallucinations and sleep paralysis. According to most authorities, diagnosis, particularly in adults, requires a combination of sleep attacks and cataplexy. The other ancillary complaints may or may not develop. All four symptoms occur in less than $50 \%$ of cases. Additional symptoms include persistent tiredness because of disrupted overnight sleep, poor memory and concentration and automatic behaviour (the result of sleepiness). Associations between narcolepsy and sleep apnoea and also REM sleep behaviour disorder (see below) are not well understood.

There have been consistent reports that many years often elapse before narcolepsy is recognised and, indeed, it seems likely that many cases are never correctly diagnosed. As each of the features mentioned above is open to misinterpretation, it is not surprising that narcolepsy is initially diagnosed as various other conditions, including neurosis, depression and personality disorder (Kryger et al, 2002). In this study, psychiatrists had made the correct diagnosis in only $11 \%$ of cases they had seen; the figure for paediatricians was $0 \%$.

The excessive sleepiness in narcolepsy (and, indeed, other pathologically sleepy conditions) can easily be misconstrued by others (and sometimes patients themselves) as laziness, boredom or depression. The sleep attacks are sometimes considered to be attention-seeking or opting-out behaviour. Cataplexy, which can occur in various forms, including episodes of localised weakness, clumsiness or merely the feeling of weakness, may be viewed as silly behaviour or even conversion disorder (Dahl, 1996). It has been reported that some cases of schizophrenia have been found to be unrecognised narcolepsy (Douglass et al, 1991).

Clinical presentations of narcolepsy can be dominated by the patient's emotional reactions to the disorder rather than its diagnostic features, especially if these are denied or concealed because of worry or embarrassment (Stores, 1999). The adverse educational, domestic, occupational and social consequences of narcolepsy (Bruck, 2001) may also serve to deflect attention from the basic condition, with patients being referred to psychiatric or, in the case of children, educational services without the cause of the problems being realised.

\section{Other primary sleep disorders characterised by pathological sleepiness}

There are other sleep disorders characterised mainly by excessive sleepiness. Idiopathic (central nervous system) hypersomnia and Kleine-Levin syndrome have the same central feature but with other associated clinical manifestations that tend to misleadingly suggest a psychiatric disorder.

\section{Idiopathic hypersomnia}

Idiopathic hypersomnia (Billiard, 1996) is an apparently rare condition but should be suspected if there is excessive daytime sleepiness, prolonged overnight sleep and great difficulty waking up in the morning (or after daytime naps) without any of the ancillary clinical features of narcolepsy or polysomnographic features of a REM sleep abnormality. The difficulty waking up is often followed by an inability to become fully alert for a prolonged period after waking. This 'sleep drunkenness' is characterised by drowsiness, confusion, disorientation, grogginess, slowness, unsteadiness, automatic behaviour and repeated returns to sleep. Sleep drunkenness can also occur in patients with sleep apnoea.

The age of onset of idiopathic hypersomnia varies from childhood to early adult life. It seems to be a lifelong disorder and, understandably, is associated with serious psychological and social problems. Treatment is said to be difficult but stimulant drugs may have some effect.

Apart from other primary sleep disorders and physical conditions causing excessive sleepiness, idiopathic hypersomnia should be distinguished from psychiatric disorders such as depression, substance misuse or volitional deficits as part of a psychiatric disorder, as well as from reluctance to engage in daytime activities, as in school refusal.

\section{Kleine-Levin syndrome}

The excessive sleepiness seen in the Kleine-Levin syndrome is intermittent (Gadoth et al, 2001). This condition seems to be rare in its full, classic form but a modified form with intermittent hypersomnia alone may be more common. The syndrome occurs mainly in males. It usually begins in puberty, often after some form of physically or psychologically stressful experience. The fully developed form consists of periods of very prolonged sleep and, when awake, overeating or hypersexual activity together with mood change and bizarre behaviour. At the end of each episode there may be a brief period of depression or elation. Episodes may last hours or weeks, each followed by return to normality for weeks or months before the next occurrence. Many cases have reported spontaneous improvement over several years. In the meantime, stimulants may be appropriate in severe cases. There are inconsistent reports of medicines, including tricyclic drugs or lithium, being helpful in preventing further attacks.

There is obvious scope for misinterpreting this syndrome as being primarily psychological in origin. 
This can cause delays in diagnosis and frustration on the part of patients and relatives (Gillberg, 1987; Pike \& Stores, 1994).

\section{Isolated sleep paralysis}

Hypnagogic (falling asleep) and hypnopompic (waking) hallucinations, and also sleep paralysis, appear to be experienced as isolated phenomena by a large proportion of the general population. Although common, their occurrence often does not come to light (even though they can be alarming) because the experiences are concealed out of embarrassment or confusion about their significance.

Sleep paralysis (Lindsley, 1992) is characterised by recurrent episodes in which ability to perform voluntary movements is lost for short periods on going to sleep or on awakening. During these episodes, communication is not possible although eye and respiratory movements remain intact and consciousness is clear. The episodes end spontaneously or with external stimulation. The condition is thought to occur occasionally in $50 \%$ or more of the population and chronically in 3-6\%. Onset is often in adolescence or early adult life. Predisposing factors include sleep disturbance and stress. The natural history is ill-defined. Serotonergic medication seems the most effective form of treatment, if required.

The paralysis may be accompanied by dramatic phenomena which are easily mistaken for a psychiatric disorder, especially as the condition is not well represented in the literature (Stores, 1998). These phenomena include simple or complex visual, auditory and somatic hallucinations (of a hypnagogic or hypnopompic type) which may lead the patient to believe that he or she is being harmed or attacked. The fear or terror engendered can be intensified by a feeling of suffocation and by a sensation of an alien presence. These universally consistent features have often been incorporated into folklore, the episodes variously being interpreted as some form of supernatural event, according to the culture.

Clearly, with these dramatic hallucinatory accompaniments and understandable anxiety on the sufferer's part, it is important to recognise sleep paralysis as a sleep disorder and not a schizophrenic or other form of psychiatric illness.

\section{REM sleep behaviour disorder}

This condition has been described relatively recently (Schenck \& Mahowald, 2002). It is of particular psychiatric interest and forensic importance. It was initially thought to be the preserve of elderly males but is now known to occur in both genders and in patients of all ages, including children. It is characterised by a pathological preservation of muscle tone in REM sleep which allows dreams to be acted out. If the dreams are violent, the patient punches, kicks, leaps or runs about. This often causes self-injury or injury to others, especially bedpartners, who may be at serious risk of harm.

Elderly males still form the majority of patients diagnosed with this condition, although women can also be affected. However, women may not come to medical attention so readily as men do, because their attacks are less aggressive. There is a strong association with neurodegenerative disorders, especially Parkinson's disease and Lewy body dementia, and also with narcolepsy. Often, a prodromal period is described (perhaps extending over many years) with persistent sleep-talking, loud vocalisations, limb twitching or gross limb and body jerking. An acute form of REM sleep behaviour disorder has been reported in association with the use of or withdrawal from alcohol or antidepressant drugs, and misuse of cocaine, amphetamines and other substances.

Because of the various links with organic factors, the condition is increasingly viewed more appropriately as a secondary parasomnia rather than the primary sleep disorder once supposed in many cases. Diagnosis rests on eliciting the typical history and demonstrating polysomnographically the REM sleep abnormalities associated with the disturbed behaviour. Clonazepam is a very effective treatment, even in the presence of neurological disease.

\section{Other sleep disorders associated with abnormal behaviour}

There are other parasomnias associated with disturbed behaviours, sometimes to a dramatic extent (Stores, 2001), either as part of the parasomnia itself or as a reaction to experiencing it. This applies to both primary parasomnias (i.e. those that are primary sleep phenomena) and secondary parasomnias (manifestations of medical or psychiatric disorders). Again, awareness of these conditions is important in order to avoid failure to recognise their sleeprelated nature. Some have medico-legal significance.

Parasomnias can occur at various stages of sleep. Some of those occurring in the pre-sleep period can be distressing or alarming to the extent that the person may dread going to bed in anticipation of experiencing the upsetting episodes.

\section{Sleep starts}

Pre-sleep hypnagogic hallucinatory phenomena may end with a sudden single jerk of the limbs or 
whole body (hypnic jerks), which can also occur separately from other experiences. They are very common and do not usually generate much concern. Less often, people describe sensory equivalents to these jerks which include intense flashes of light or a loud bang, crack or snapping noise (for which the term 'exploding head syndrome' has been coined by Sachs \& Svanborg, 1991). Other alarming experiences of a similar type include sudden pain or other unpleasant sensations. Not surprisingly, those having such sensory 'sleep starts' imagine that they are at risk of some cerebral catastrophe and need reassurance that the episodes are, in fact, essentially benign.

\section{Restless legs syndrome}

Restless legs syndrome is another condition occurring mainly in the pre-sleep period. It consists of various disagreeable leg sensations with an irresistible urge to move the legs. Although not usually the case, it can be associated with pregnancy or physical illness such as uraemia. In severe forms, it can be extremely distressing to the sufferer and also the bed-partner. Periodic limb movements in sleep (capable of impairing the restorative value of sleep) often accompany the syndrome.

\section{Sleeproalking/sleep terrors}

It is often not appreciated that complicated behaviour can be enacted while a person is still asleep. Failure to recognise this fact can lead to erroneous psychiatric conclusions and, in legal cases, the mistaken view that a person was aware of his or her actions and therefore responsible for them. Sleepwalking is the main example of this. Predominantly a childhood sleep disorder, it is said to occur in perhaps 3\% of adults, sometimes frequently (Hublin et al, 1997). Sleepwalking seems to be the result of a genetic predisposition to abnormal arousal from deep non-REM sleep, but episodes can be provoked by an increase in deep sleep (including that caused by sleep loss, sedative medications or alcohol) and sleep-disturbing factors, including stress. Typically, episodes occur early in overnight sleep. A wide range of behaviours combine features of apparently being awake yet remaining asleep. The sleepwalker may be calm but confused and can behave inappropriately in various ways. Alternatively, agitation and even frenzy (as if trying to escape from some danger) dominate the picture. Such behaviour shades into the clinical manifestations of the related and often coexistent condition of sleep (night) terrors.

During sleep, complicated actions are possible if they are habitual or familiar, e.g. making meals, travelling along routes outside the house or even driving a car. Behaviour during sleepwalking can result in accidental personal injury or injury to others from violent and even homicidal behaviour, although this is rare. Sexual behaviour during sleep (Rosenfeld \& Elhajjar, 1998) is a further source of legal debate about responsibility for actions during sleepwalking or related sleep states. Guidelines have been suggested to help identify sleep-related criminal acts which have a wide differential diagnosis and include psychogenic dissociative states, post-traumatic stress disorder and malingering (Mahowald \& Schenck, 1999).

\section{Overlap parasomnia disorder}

Mahowald \& Schenck (1992) have emphasised the existence of complicated sleep disorders (including a number which could be misconstrued as psychiatric conditions) that cross the traditional boundaries between sleep and wakefulness or between the traditionally recognised parasomnias. An example of the latter is 'overlap parasomnia disorder', in which patients manifest a combination of sleepwalking, sleep terrors and REM sleep behaviour disorder for which psychiatric treatment is often given before the correct diagnosis is made (Schenck et al, 1997).

\section{Other parasomnias}

Other parasomnias that have been described comparatively recently are also at risk of being misconstrued as primarily psychiatric disorders, mainly because of unfamiliarity with their existence and nature. Sleep-related eating disorders illustrate how strange behaviour at night can be a feature of various underlying sleep disorders such as sleepwalking, OSAS and narcolepsy (Winkelman et al, 1999). The clinical features of the seizures in nocturnal frontal lobe epilepsy (Provini et al, 2000) are often so bizarre and far removed from the usual concept of an epileptic attack that this form of epilepsy is often thought to be a serious psychiatric disorder. Features can include kicking, hitting, rocking, thrashing and cycling or scissor movements of the legs, sometimes accompanied by alarming vocalisations such as shouting, screaming or roaring. Other forms of sleep-related epilepsy can be misconstrued in a similar way (Autret et al, 1999).

\section{Conclusions}

Sleep disturbance is intimately linked with both child and adult psychiatric disorders in a number of ways. Of basic clinical importance is the fact that many specific sleep disorders are at risk of being 
misconstrued as primarily psychiatric conditions and, as a consequence, treated inappropriately.

More detailed enquiries about sleep than are usual in traditional history-taking would be a first step in helping psychiatrists to recognise sleep disorder in patients and identify its nature. Basic screening questions that should be asked routinely of patients and observers are whether the patient has difficulty getting to sleep or staying asleep, whether excessive sleepiness is a problem during the day and whether the patient has any disturbing episodes at night. A positive answer to any of these questions calls for a detailed sleep history (Aldrich \& Naylor, 2000). A sleep-wake questionnaire can also be a useful part of clinical enquiry (e.g. Roth et al, 2002). By means of detailed information collected in these ways, together with an overall review of the patient's past history and his or her physical and psychiatric condition, it should be possible to identify, at least provisionally, the nature of any sleep disorder present, provided that the clinician understands the range of disorders described in modern sleep disorders medicine and their main features. Depending on these preliminary findings, further special investigations, including psychological sleep studies, might be appropriate, with the advice of a sleep disorders specialist in selected cases.

It is essential that sleep disorders do not continue to be overlooked or misinterpreted, not only in psychiatry but in other medical specialities concerned with the care of patients of all ages. Inevitably, the consequences of not recognising the patient's basic condition and failing to provide appropriate treatment has serious consequences for patients and their families.

\section{References}

*Aldrich, M. S. \& Naylor, M. W. (2000) Approach to the patient with disordered sleep. In Principles and Practice of Sleep Medicine (3rd edn) (eds M. H. Kryger, T. Roth \& W. C. Dement), pp. 521-525. Philadelphia, PA: Saunders.

American Sleep Disorders Association (1997) ICSD International Classification of Sleep Disorders, Revised: Diagnostic and Coding Manual. Rochester, MN: American Sleep Disorders Association.

Autret, A., de Toffo, I. B., Corcia, P. L., et al (1999) Sleep and epilepsy. Sleep Medicine Reviews, 3, 201-217.

*Benca, R. M. (2000) Psychiatric sleep-wake disorders. In New Oxford Textbook of Psychiatry (eds M. G. Gelder, J. J. López-Ibor \& N. C. Andreason), vol. 1, pp. 1021-1026. Oxford: Oxford University Press.

Billiard, M. (1996) Idiopathic hypersomnia. Neurologic Clinics, 14, 573-582.

Bliwise, D. L. (1996) Is sleep apnea a cause of reversible dementia in old age? Journal of the American Geriatric Society, 44, 1287-1294.

*Bonnet, M. H. (2000) Sleep deprivation. In Principles and Practice of Sleep Medicine (3rd edn) (eds M. H. Kryger, T. Roth \& W. C. Dement), pp. 53-71. Philadelphia, PA: Saunders.

Bruck, D. (2001) The impact of narcolepsy on psychological health and role behaviours: negative effects and comparisons with other illness groups. Sleep Medicine, 2, 437-446.
Brunello, N., Armitage, R., Feinberg, I., et al (2000) Depression and sleep disorders: clinical relevance, economic burden and pharmacological treatment. Biological Psychiatry, 42, 107-119.

Dagan, Y. (2002) Circadian rhythm sleep disorder (CRSD). Sleep Medicine Reviews, 6, 45-55.

Dahl, R. E. (1996) Narcolepsy in children and adolescents. Child and Adolescent Psychiatric Clinics of North America, 5, 649-659.

Douglass, A. B., Hays, P., Pazderka F., et al (1991) Florid refractory schizophrenias that turn out to be treatable variants of HLA-associated narcolepsy. Journal of Nervous and Mental Disease, 179, 12-17.

Engelman, H. M., Kingshott, R. N., Martin, S. E., et al (2002) Cognitive functions in the sleep apnea/hypopnea syndrome (SAHS). Sleep, 23 (suppl. 1), S102-S108.

*Fallone, G., Owens, J. A. \& Deane, J. (2002) Sleepiness in children and adolescents: clinical implications. Sleep Medicine Reviews, 6, 287-306.

*Ford, D. E. \& Kamerow, D. B. (1989) Epidemiologic study of sleep disturbances and psychiatric disorders. An opportunity for prevention? JAMA, 262, 1479-1484.

Gadoth, N., Kesler, A., Vainstein, G., et al (2001) Clinical and polysomnographic characteristics of 34 patients with Kleine-Levin syndrome. Journal of Sleep Research, 10, 337341.

Gillberg, C. (1987) Kleine-Levin syndrome: unrecognized diagnosis in adolescent psychiatry. Journal of the American Academy of Child and Adolescent Psychiatry, 26, 793-794.

Gillin, J. C. (1998) Are sleep disturbances risk factors for anxiety, depression and addictive disorders. Acta Psychiatrica Scandinavica Supplementum, 393, 39-43.

Howland, R. H. (1997) Sleep-onset rapid eye movement periods in neuropsychiatric disorders: implications for the pathophysiology of psychosis. Journal of Nervous and Mental Disease, 185, 730-738.

Hublin, C., Kaprio, J., Partinen, M., et al (1997) Prevalence and genetics of sleepwalking: a population-based twin study. Neurology, 48, 177-181.

*Katz, D. A. \& McHorney, C. A. (2002) The relationship between insomnia and health-related quality of life in patients with chronic illness. Journal of Family Practice, 51, 229-235.

Kryger, M. H., Walid, R. \& Manfreda, J. (2002) Diagnoses received by narcolepsy patients in the year prior to diagnosis by a sleep specialist. Sleep, 25, 36-41.

Lindsley, J. G. (1992) Sleep paralysis. In Movement Disorders in Neurology and Neuropsychiatry (eds A. B. Joseph \& R. G. Young), pp. 602-619. Boston, MA: Oxford University Press.

Mahowald, M. W. \& Schenck, C. H. (1992) Dissociated states of wakefulness and sleep. Neurology, 42 (suppl. 6), $44-52$.

* _ \& - (1999) Medico-legal aspects of sleep medicine. Neurologic Clinics, 17, 215-234.

Naseem, S., Chaudhary, B. \& Collop, N. (2001) Attention deficit hyperactivity disorder in adults and obstructive sleep apnea. Chest, 119, 294-296.

*Obermeyer, W. H. \& Benca, R. M. (1996) Effects of drugs on sleep. Neurologic Clinics, 14, 827-840.

Ohayon, M. M., Guilleminault, C., Priest, R. G., et al (1997) Snoring and breathing pauses during sleep: telephone interview of a United Kingdom population sample. BMJ, 314, 860-863.

Pike, M. \& Stores, G. (1994) Kleine-Levin syndrome: a cause of diagnostic confusion. Archives of Disease in Childhood, 71, 355-357.

Provini, F., Plazzi, G., Montagna, P., et al (2000) The wide clinical spectrum of nocturnal frontal lobe epilepsy. Sleep Medicine Reviews, 4, 375-386.

Regenstein, Q. R. \& Monk, T. H. (1995) Delayed sleep phase syndrome: a review of its clinical aspects. American Journal of Psychiatry, 152, 602-608.

Rosenfeld, D. S. \& Elhajjar, A.-J. (1998) Sleep sex: a variant of sleepwalking. Archives of Sexual Behaviour, 27, $269-278$. 
Roth, T., Zammit, G., Kushida, C., et al (2002) A new questionnaire to detect sleep disorders. Sleep Medicine, 3 99-108.

Sachs, C. \& Svanborg, E. (1991) The exploding head syndrome: polysomnographic recordings and therapeutic suggestions. Sleep, 14, 263-266.

Schenck, C. H. \& Mahowald, M. W. (2002) REM sleep behavior disorder: clinical, developmental, and neuroscience perspectives 16 years after its formal identification in Sleep. Sleep, 25, 120-138.

—, Boyd, J. L. \& Mahowald, M. W. (1997) A parasomnia overlap disorder involving sleepwalking, sleep terrors, and REM sleep behavior disorder in 33 polysomnographically confirmed cases. Sleep, 20, 972-981.

Smith, R., Ronald, J., Delaive, K., et al (2002) What are obstructive sleep apnea patients being treated for prior to this diagnosis? Chest, 121, 164-172.

Stores, G. (1998) Sleep paralysis and hallucinosis. Behavioural Neurology, 11, 109-112.

- (1999) Recognition and management of narcolepsy. Archives of Disease in Childhood, 81, 519-524.

- (2001) Dramatic parasomnias. Journal of the Royal Society of Medicine, 94, 173-176.

- \& Wiggs, L. (2001) Sleep Disturbance in Children and Adolescents with Disorders of Development: Its Significance and Management (Clinics in Developmental Medicine No. 155). London: Mac Keith.

Tanskanen, A., Tuomilehto, J., Viinamäki, H., et al (2001) Nightmares as predictors of suicide. Sleep, 24, 844-847.

Thorpy, M. (2001) Current concepts in the etiology, diagnosis and treatment of narcolepsy. Sleep Medicine, 2, 5-17.

*Wagner, D. R. (1990) Circadian rhythm sleep disorders. In Handbook of Sleep Disorders (ed. M. J. Thorpy), pp. 493527. New York: Dekker.

Walters, A. S., Mandelbaum, D. E., Lewin, D. S., et al (2000) Dopaminergic therapy in children with restless legs/ periodic limb movements in sleep and ADHD. Dopaminergic Therapy Study Group. Pediatric Neurology, 22, 182186.

Weissman, M. M., Greenwald, S., Niño-Murcia, G., et al (1997) The morbidity of insomnia uncomplicated by psychiatric disorders. General Hospital Psychiatry, 19, 245-250.

Winkelman, J. W., Herzog, D. B. \& Fava, M. (1999) The prevalence of sleep-related eating disorder in psychiatric and non-psychiatric populations. Psychological Medicine, 29, 1461-1466.

* indicates publications of particular interest.

\section{Further reading}

Kryger, M. H., Roth, T. \& Dement, W. C. (2000) Principles and Practice of Sleep Medicine (3rd edn). Philadelphia, PA: WB Saunders.

Stores, G. (2001) A Clinical Guide to Sleep Disorders in Children and Adolescents. Cambridge: Cambridge University Press.

\section{Multiple choice questions}

1 Common features of delayed sleep phase syndrome include:

a difficulty getting off to sleep

b daytime tiredness, fatigue and depression

c adolescent disputes with parents

$\mathrm{d}$ all of the above

e none of the above.

2 Obstructive sleep apnoea syndrome:

a does not occur in women

b is linked with cardiovascular disease

c can produce a picture of dementia

$\mathrm{d}$ is often untreatable

e usually comes readily to medical attention.

\section{Narcolepsy:}

a usually involves cataplexy

b is usually correctly diagnosed by psychiatrists

c always includes sleep paralysis

d may involve hallucinatory experiences

e does not occur in children.

4 REM sleep behaviour disorder:

a is basically a disorder of non-REM sleep

b only occurs in elderly males

c is generally untreatable

d may precede the onset of dementia

e is difficult to diagnose clinically.

5 Sleepwalking episodes:

a can involve complicated actions

b often run in families

c tend to occur later in the night

d may cause accidental injury to others

e can be provoked by alcohol.

\section{MCQ answers}

$\begin{array}{llllllllll}1 & & 2 & & 3 & & 4 & & 5 & \\ \text { a } & \text { T } & \text { a } & \text { F } & \text { a } & \text { T } & \text { a } & \text { F } & \text { a } & \text { T } \\ \text { b } & \text { T } & \text { b } & \text { T } & \text { b } & \text { F } & \text { b } & \text { F } & \text { b } & \text { T } \\ \text { c } & \text { T } & \text { c } & \text { T } & \text { c } & \text { F } & \text { c } & \text { F } & \text { c } & \text { F } \\ \text { d } & \text { T } & \text { d } & \text { F } & \text { d } & \text { T } & \text { d } & \text { T } & \text { d } & \text { T } \\ \text { e } & \text { F } & \text { e } & \text { F } & \text { e } & \text { F } & \text { e } & \text { F } & \text { e } & \text { T }\end{array}$

